\title{
Evolutionary origin of highly repetitive plastid genomes within the clover genus (Trifolium)
}

Saemundur Sveinsson ${ }^{*}$ and Quentin Cronk

\begin{abstract}
Background: Some clover species, particularly Trifolium subterraneum, have previously been reported to have highly unusual plastomes, relative to closely related legumes, enlarged with many duplications, gene losses and the presence of DNA unique to Trifolium, which may represent horizontal transfer. In order to pinpoint the evolutionary origin of this phenomenon within the genus Trifolium, we sequenced and assembled the plastomes of eight additional Trifolium species widely sampled from across the genus.

Results: The Trifolium plastomes fell into two groups: those of Trifolium boissieri, T. strictum and T. glanduliferum (representing subgenus Chronosemium and subg. Trifolium section Paramesus) were tractable, assembled readily and were not unusual in the general context of Fabeae plastomes. The other Trifolium species ("core Trifolium") proved refractory to assembly mainly because of numerous short duplications. These species form a single clade, which we call the "refractory clade" (comprising subg, Trifolium sections Lupinaster, Trifolium, Trichocephalum, Vesicastrum and Trifoliastrum). The characteristics of the refractory clade are the presence of numerous short duplications and 7-15\% longer genomes than the tractable species. Molecular dating estimates that the origin of the most recent common ancestor (MRCA) of the refractory clade is approximately 13.1 million years ago (MYA). This is considerably younger than the estimated MRCA ages of Trifolium (c. 18.6 MYA) and Trifolium subg. Trifolium (16.1 MYA).

Conclusions: We conclude that the unusual repetitive plastome type previously characterized in Trifolium subterraneum had a single origin within Trifolium and is characteristic of most (but not all) species of subgenus Trifolium. It appears that an ancestral plastome within Trifolium underwent an evolutionary change resulting in plastomes that either actively promoted, were permissive to, or were unable to control, duplications within the genome. The precise mechanism of this important change in the mode and tempo of plastome evolution deserves further investigation.
\end{abstract}

Keywords: Trifolium, Clover, Plastome evolution, Plastid genome evolution, Repetitive DNA, Fabaceae

\section{Background}

The increased availability and lowered costs of various massive parallel sequencing technologies have resulted in a dramatic expansion of fully sequenced plastid genomes [1]. There are currently 512 plastome sequences listed at NCBI's Organelle Genome Resource website (http://tinyurl.com/ncbi-plastid-genomes, 17 April 2014) and half of them have been made available since 2012. Their structure, gene order and gene content is generally highly conserved across most flowering plants, where most plastomes have two copies of a highly conserved

\footnotetext{
* Correspondence: saemundur.sveinsson@gmail.com

Department of Botany and Biodiversity Research Centre, University of British Columbia, 6270 University Boulevard, Vancouver, BC V6T 1Z4, Canada
}

inverted repeat (IR) and two conserved single copy regions (see [2]). However, there are some well known and striking exceptions from these conserved plastome structures in several photosynthetic angiosperm lineages, such as the Geraniaceae [3] and Campanulaceae [4].

Considerable advances have recently been made in the development of genomic resources for the clover genus (Trifolium), with the publication of the genome assembly [5] and analyses of the transcriptome [6] of red clover (T. pratense). Subterranean clover (Trifolium subterraneum) is known to have an unusual plastid genome structure [7]. Firstly it lacks one copy of the inverted repeat, but that is a character shared with a large group of papilionoid legumes [8], designated the inverted repeat 
lacking clade (IRLC). Secondly its plastome has undergone more than a dozen rearrangements, i.e. translocations and/or inversions, compared to the plastid genome of Medicago truncatula [7]. However, highly rearranged plastomes also occur in the related tribe Fabeae, represented by Lathyrus sativus and Pisum sativum [9]. Finally, and very unusually, its plastome contains about fourfold the amount of repeated DNA compared to related legume species and is consequently about $20 \mathrm{~kb}$ longer [7]. A recent study has shown that this unusual repeat-rich plastome structure is not unique to T. subterraneum [10]. However, the extent to which this is a widespread feature of Trifolium species, or characteristic of a restricted part of the genus Trifolium still needs further investigation. To answer this question, we performed a low coverage whole genome shotgun sequencing of nine strategically sampled Trifolium species and were able to assemble eight plastid genomes. These plastomes were then analysed to elucidate the phylogenetic distribution of plastome variation in the genus.

\section{Results}

\section{Plastome assembly and structural variability}

The plastomes of three sequenced Trifolium species, Trifolium boissieri, T. strictum and T. glanduliferum, were tractable, assembled easily and had no unusual structure, at least in the context of related species in the inverted repeat loss clade (IRLC), such as species of the tribe Fabeae (see [9,10]) (Figure 1A and C) [GenBank: KJ788284, KJ788292 and KJ788285]. The remaining six Trifolium species had plastome structure similar to $T$. subterraneum (described in [7]), containing several short repetitive regions, which made them refractory to assembly (see Table 1). However, with one exception we were able to assemble all of them successfully, using careful analysis of the paired-end sequences (see below) (Figure 1B and D) [GenBank: KJ788286 - KJ788289 and KJ788291]. The exception is $T$. pratense. The plastome structure of $T$. pratense appears to be highly complex and we were unable to complete a full assembly of it using the methods employed in this study. It is clear that this plastome contains several repeated regions, similar to T. subterraneum. We were, however, able to put together three large plastid contigs for $T$. pratense, with a combined length of $121 \mathrm{~kb}$, which were annotated with DOGMA [11] and used for phylogenetic analysis [GenBank: KJ788290]. We generated well supported plastome assemblies for the remaining five "difficult" species and verified them using mapping information from the paired end Illumina reads (see Table 1 ). We ensured that the entire assembly had sufficient coverage (at least $100 \mathrm{x}$ ) and that the placement of adjacent plastid regions were supported by the mapping of paired end reads. However it is important to note, that despite these careful quality checks, assembly of some regions that have a particularly high frequency of repeats should remain provisional. Consequently these are deposited on GenBank as "unverified".

\section{Phylogenetic distribution of the refractory species}

The six Trifolium species with plastomes that were refractory to assembly differed markedly from the three species that were tractable for assembly. Furthermore, the six species and the previously sequenced T. subterraneum and $T$. repens all belong to the same clade within Trifolium, which we call the "refractory clade", comprising subg. Trifolium sections Lupinaster, Trifolium, Tricocephalum, Vesicastrum and Trifoliastrum (see Figure 2).The remaining three species, which have plastomes that assembled readily, are all outside this clade and represent subgenus Chronosemium and subgenus Trifolium section Paramesus (see Figure 2). Molecular dating analysis of the refractory clade revealed that it originated, i.e. had a most recent common ancestor (MRCA), about 12.4 - 13.8 MYA. The refractory clade has therefore had a considerable amount of time in which to accumulate a high diversity of repeat patterns. This compares to estimated MRCA ages of 16.1 MYA for Trifolium subgenus Trifolium and 18.4 MYA for the genus Trifolium as a whole. The phylogeny in Figure 2 was inferred using maximum likelihood from a concatenated matrix of 68 protein coding plastid genes, with a combined aligned length of just over $48 \mathrm{~kb}$. All nodes have $100 \%$ bootstrap support and the topology is consistent with the most recent phylogenetic treatment of Trifolium [12].

\section{The nature of the duplicated regions}

The amount of repetitive sequence in the Trifolium plastomes ranged from $0.71 \%$ to $20.71 \%$ (see Table 1). This was based on a reciprocal BLASTN search, where each plastome was used as the query and subject (see Methods). Not surprisingly, plastomes belonging to the refractory clade contained about 5-20 times more repetitive DNA than Trifolium species in subgenus Chronosemium and subgenus Trifolium section Paramesus. Repetitive regions larger than $300 \mathrm{bp}$ were subjected to a BLASTX search, in order to determine whether they contained any protein coding regions. A protein database was compiled of all annotated genes from the Trifolium plastomes. About $60 \%$ of the longer repetitive regions did not show significant (E-value $\leq 1 \mathrm{E}-06)$ similarity to any protein coding regions. The remaining $40 \%$ of showed similarity to one of the following plastid genes: clpP, psaJ, psbK, psbN, rpl2, rpl23, rpoB, rps18, $r p s 3, y c f 1$ and $y c f 3$, indicating that at least partial duplication of genic regions has occurred. Patterns of genic duplication are highly variable, and no genes were 


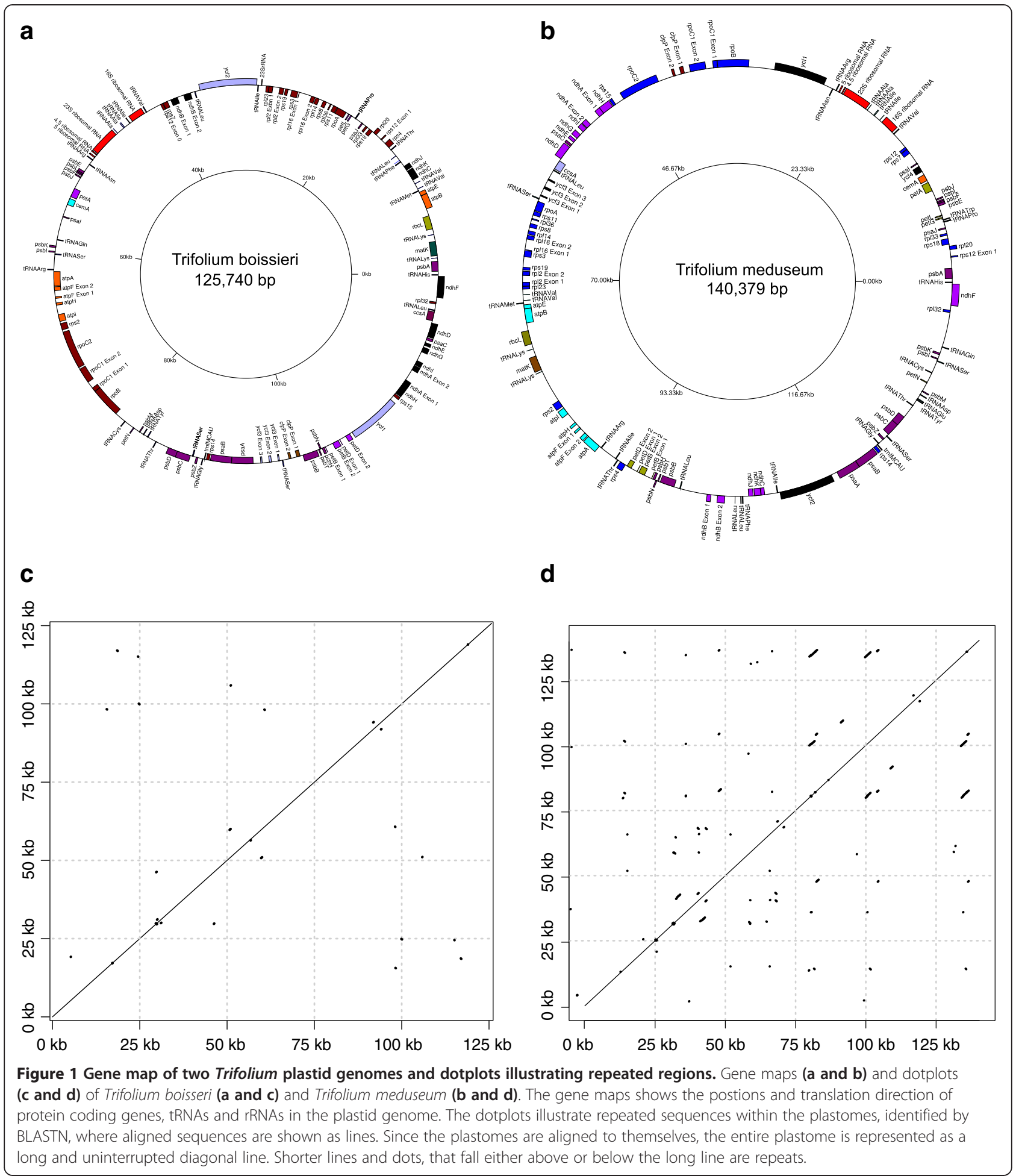

found to be duplicated in more than two species. All repetitive gene regions consisted of only a partial reading frame and no duplicated genes were found with the entire reading frame intact in more than one copy. However a small number of gene duplicates appeared to have relatively large portions of open reading frames
(ORF) that appeared to be intact (i.e. identical at the protein level to the full length functional copy). A BLASTX search of repetitive regions revealed that $p s a J$, $p s b K, p s b N, r p l 2, y c f 1$ and $y c f 3$ had instances where they are $100 \%$ intact over $5-30 \%$ of the length of the corresponding complete ORF. 
Table 1 Summary Illumina sequencing accessions and plastome assembly information of Trifolium species

\begin{tabular}{|c|c|c|c|c|}
\hline $\begin{array}{l}\text { Species (USDA seed } \\
\text { accession) [Section] }\end{array}$ & $\begin{array}{l}\text { NO trimmed reads } \\
\text { (mean length) [SD*] }\end{array}$ & $\begin{array}{l}\text { Plastome length } \\
\text { (repetitive \%) }\end{array}$ & $\begin{array}{l}\text { GenBank } \\
\text { accession }\end{array}$ & $\begin{array}{l}\text { Herbarium } \\
\text { voucher }\end{array}$ \\
\hline Medicago truncatula & NA & $124,033 \mathrm{nt}$ & NC_003119 & NA \\
\hline Gaertn. (NA) [NA] & & $(1.46 \%)$ & & \\
\hline T. aureum Pollich (NA) & NA & 126,970 & NC_024035 & NA \\
\hline [Subg. Chronosemium] & & $\left(5.6 \%{ }^{* *}\right)$ & & \\
\hline T. grandiflorum Schreb. (NA) & NA & 125,628 & NC_024034 & NA \\
\hline [Subg. Chronosemium] & & $\left(4.3 \%^{* *}\right)$ & & \\
\hline T. subterraneum L. & NA & $144,763 \mathrm{nt}$ & NC_011828 & NA \\
\hline (NA) [Trichocephalum] & & $(20.71 \%)$ & & \\
\hline T. repens $L$. (NA) & NA & 132,120 & NC_024036 & NA \\
\hline [Trifoliastrum] & & $\left(20.7 \%^{* *}\right)$ & & \\
\hline Trifolium boisseri Guss. (PI 369022) & $20.5 \times 10^{6}$ & $125,741 \mathrm{nt}$ & KJ788284* & Sveinsson 14-01 (UBC) \\
\hline [Subg. Chronosemium] & $(95.02)[14.08]$ & $(1.05 \%)$ & & \\
\hline T. strictum L. & $16.7 \times 10^{6}$ & $125,835 \mathrm{nt}$ & KJ788292* & Sveinsson 14-02 (UBC) \\
\hline (PI 369147) [Paramesus] & (95.13) [13.98] & $(0.71 \%)$ & & \\
\hline T. glanduliferum Boiss. (PI 296666) & $11.5 \times 10^{6}$ & $126,182 \mathrm{nt}$ & KJ788285* & Sveinsson 14-03 (UBC) \\
\hline [Paramesus] & (95.16) [13.96] & $(0.78 \%)$ & & \\
\hline T. lupinaster L. & $24.8 \times 10^{6}$ & $135,077 \mathrm{nt}$ & KJ788287* & Sveinsson 14-04 (UBC) \\
\hline (PI 631632) [Lupinaster] & $(94.77)[14.33]$ & (5.98\%) & & \\
\hline T. meduseum Blanche & $18.9 \times 10^{6}$ & $140,380 \mathrm{nt}$ & KJ788288* & Sveinsson 14-05 (UBC) \\
\hline ex Boiss. (PI 369049) [Trichocephalum] & $(95.14)[14.01]$ & $(12.83 \%)$ & & \\
\hline T. pratense L. CV. & $22.2 \times 10^{6}$ & NA & KJ788290* & Sveinsson 14-07 (UBC) \\
\hline Arlington (G 27569) [Trifolium] & $(94.85)[14.29]$ & (NA) & & \\
\hline T. hybridum L. & $21.4 \times 10^{6}$ & $134,881 \mathrm{nt}$ & KJ788286* & Sveinsson 14-08 (UBC) \\
\hline $\begin{array}{l}\text { (PI 634109, as T. pallescens) } \\
\text { [Vesicastrum] }\end{array}$ & (95.11) [14.04] & $(7.86 \%)$ & & \\
\hline T. semipilosum & $29.1 \times 10^{6}$ & $138,242 \mathrm{nt}$ & KJ788291* & Sveinsson 14-09(UBC) \\
\hline Fresen. (PI 262238) [Vesicastrum] & (94.80) [14.33] & $(10.55 \%)$ & & \\
\hline T. occidentale Coombe & $18.6 \times 10^{6}$ & $133,806 \mathrm{nt}$ & KJ788289* & Sveinsson 14-10 (UBC) \\
\hline (PI 641363) [Trifoliastrum] & (95.35) [13.80] & $(4.64 \%)$ & & \\
\hline
\end{tabular}

An asterisk marks plastome sequences newly reported in this paper. Only a partial assembly of $T$. pratense was possible with our data (see text for explanation). *SD: Standard Deviation.

**Estimations from [10].

\section{Instances of gene loss}

The Trifolium plastomes have undergone some gene loss. AccD appears to missing from plastomes in the following sections of the subgenus Trifolium: Trifolium, Tricocephalum, Vesicastrum and Trifoliastrum. This is in agreement with previous reports $[7,10]$. Our broad phylogenetic sampling shows that the plastid copy of $a c c D$ is also still intact in Trifolium lupinaster (section Lupinaster of the subgenus Trifolium).

\section{Discussion}

\section{The Phylogenetic distribution of plastome types}

Two types of plastomes were observed among the Trifolium species in this study. Three species had no unusual structure in the context of IRLC plastomes, which are known to have numerous rearrangements (see $[9,10]$ ). The six remaining species had enlarged, repeatcontaining plastome structure similar to $T$. subterraneum and $T$. repens (described in $[7,10]$ ). These had several repeated regions and were $7-21 \mathrm{~kb}$ larger compared to Medicago truncatula, T. boissieri, T. strictum and T. glanduliferum (see Figure 2). Furthermore, we observed a strong phylogenetic clustering of these larger, more repeated plastomes. They comprise a single clade (Figure 2) and, strikingly, all the sampled species of this clade have the unusual repeat-rich plastomes. The North-American section Involucrarium also belongs to this clade (it is sister to section Trifoliastrum [12]), 


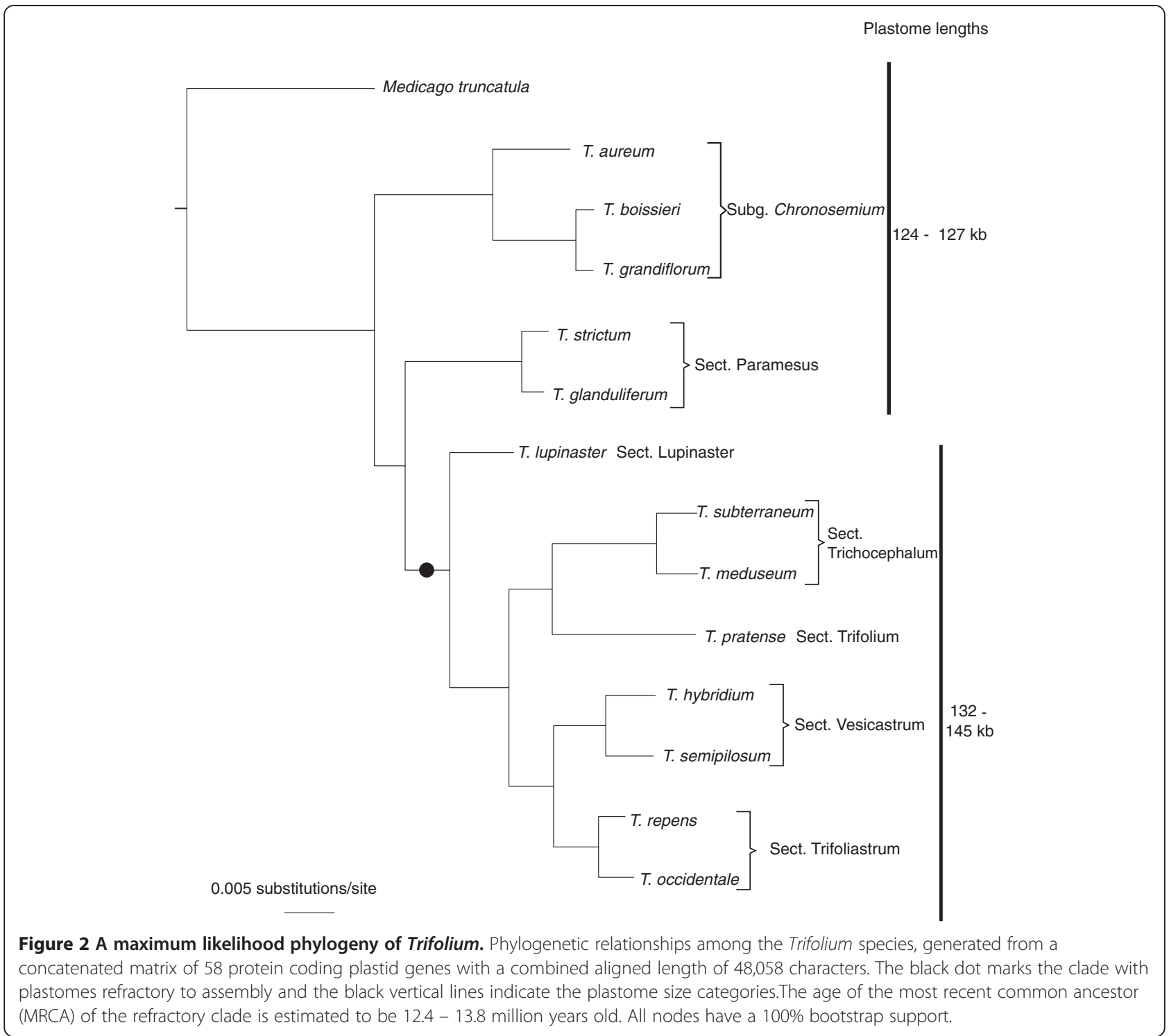

and is therefore likely to have similar "refractory" plastomes, although this has yet to be confirmed. This clade contains about 200 species or roughly $70 \%$ of the genus, which has a total of roughly 300 species. The evolutionary success of the clade with the repetitive plastomes leaves no doubt about the functional effectiveness of these plastomes despite their unusual structure.

\section{Potential causes of genome instability and functional significance of the repeat regions}

Some regions within the T. subterraneum plastome have been suspected to be of bacterial origin by lateral transfer [7], however those regions are not repetitive. The repetitive regions seem to be of plastid origin, as our BLASTN and BLASTX searches to NCBI's nucleotide collection (nt/nr) and non-redundant protein sequences (nr), did not indicate any obvious non-plastid sequence. A more recent study found no evidence of any bacterial DNA in plastid genomes of several Trifolium species [10]. Repeated sequences have been associated with the plastid rearrangements in other angiosperm lineages, such as the Campanulaceae $[4,13]$ and Geraniaceae $[3,14]$. However, it is clear that plastomes can be highly rearranged without being highly repetitive, such as Pisum sativum and Lathyrus sativus (Fabaceae) [9].

It is not yet known what has caused repeated sequences to evolve within the plastomes of the Trifolium refractory clade although it is likely that this is under the control of nuclear genes. Plastid reorganization is a common process and there is evidence that reorganized molecules may comprise around $1 \%$ of the plastid complement of plant cells [15]. This variation may be caused 
by either homologous recombination (HR) acting on perfect repeats of $>50 \mathrm{bp}$, or microhomology-mediated break-induced replication (MMBIR) acting on microhomologous repeats of $<30$ bp [16,17]. Various nuclear genes are known to be important in maintaining plastome stability through recombination control and surveillance, and these are therefore candidate genes for genome instability in certain lineages. The $r e c A$ gene has an important role in homologous recombination and there are plant copies that localize to the chloroplast $[18,19]$. Plastid-targeted WHIRLY genes are known to promote plastome stability, apparently by preventing build up of abnormal molecules produced by MMBIR. Arabidopsis plants lacking functional copies of relevant AtWHY genes show increased accumulation of abnormal plastid DNA with irregular duplications, deletions and circularization events [16].

Most of these abnormal plastid forms are deleterious and transient, not contributing to plastid evolution in the majority of plant lineages. An important consideration therefore is not the genomic changes that cause an increased frequency of plastome reorganization, but what genome changes allow such major plastome changes to persist without being eliminated as deleterious. It may be that certain plastid genes are particularly sensitive to genome rearrangements and their removal to the nuclear genome makes the plastid genome more permissive to rearrangement. The plastid gene $a c c D$ for instance, known to be essential for plant development [20] and with recombinationally active repeats [21], has been moved to the nucleus independently in two lineages with highly rearranged plastomes: Campanulaceae [22] and Trifolium $[9,10]$.

One possible consequence of having repeated sequences in a circular molecule, such as the plastid genome, is that if recombinationally active they could potentially allow intramolecular recombination of the plastome into subgenomic molecules much in the manner of the plant mitochondrion, which is rich in recombinational repeats. This has been suggested as a reason for the repeat richness of certain algal plastomes [23]. The Trifolium plastomes are much less rich in repeats than Chlamydomonas, or than the mitochondrial genome of land plants, but it is nevertheless interesting to consider the idea that the repeat accumulation in Trifolium plastomes is being driven by the advantage of maintaining substochiometric populations of specific ORFs in cellular subpopulations [24].

\section{Conclusions}

Although we have sampled only a relatively small number of species in a large genus, the results are nevertheless striking. We show that the $T$. subterraneum-type plastome, i.e. containing large amounts of repetitive DNA, is phylogenetically restricted to a single core clade of the genus (the "refractory clade"), comprising five of the eight recognized sections within Trifolium. Furthermore it is ubiquitously present in all members of this clade in our sample. It is thus reasonable to suppose that this may be the characteristic plastome of this core clade. Trifolium is a large genus of c. 300 species, and these five sections contain slightly over 200 species, or about $70 \%$ of the genus. There are therefore likely to be abundant exemplars of this unusual plastome type, at varying degrees of evolutionary relatedness, available for future functional and evolutionary studies.

\section{Methods}

\section{Plant material and Illumina sequencing}

Sampling was strategically placed across the genus using the sectional classification of [12]. Total DNA was extracted from fresh leaf material of plants that had been grown from seeds in a greenhouse (at UBC), following a modified version of the CTAB protocol [25]. The seeds were obtained from the United States Department of Agriculture (USDA) National Plant Germplasm System, more specifically the National Temperate Forage Legume Genetic Resources Unit in Prosser, WA. Plants were grown until they flowered, the material was critically determined and herbarium specimens collected (of all but one accession, see Table 1). RNase treatments were performed (cat. 19101, QIAGEN, Germantown, MD) and DNA quality was assessed by visual inspections on $1 \%$ agarose gels. Illumina sequencing libraries were constructed from high quality DNA, using the NEXTflexTM DNA sequencing kit (100 bp Paired-End reads) (cat: 5140-02, Bioo Scientific Corp, TX). We followed the manufacturer's protocol and c. 400 bp DNA fragments were size selected using Agencourt AMPure XpTM magnetic beads (cat. A63880, Beckman Coulter Genomics, MA). Completed libraries were pooled and sequenced on a lane of the Illumina HiSeq-2000 platform.

\section{Plastid genome assemblies and annotation}

Trimmomatic v.0.3 [26] was used to trim and remove low quality Illumina reads, with the following flags: LEADING:20 TRAILING:20 SLIDINGWINDOW:4:15MINLEN:36. High quality reads were used in all subsequent analysis and singlet reads, i.e. reads without a paired end, were discarded. We used the de novo method implemented in CLC Genomic Workbench v.7.0.2 to generate assemblies for each species, using the default settings. Contigs of plastid origin were identified by a blast search [27] to the available plastid genome of Trifolium subterraneum, published in [7] [GenBank: NC_011828]. These were generally the largest and most highly covered contigs in the de novo assembly and always had an E-value of 0 when blasted 
to the T. subterraneum plastome. Regions with nucleotides represented as Ns were manually resolved by retrieving sequence information directly from the quality trimmed reads. For three out of eight species, the de novo assembly returned a single large plastid contig. This was not the case for the plastid genome assembly of the remaining six Trifolium species, where the de novo assembly resulted in about five to 10 plastid contigs. In those cases we decided to extend both ends of the plastid contigs by manually appending sequence from the Illumina reads. The sequence of each contig was extended until an overlap of at least $50 \mathrm{bp}$ could be identified at the end of another contig in the assembly, whereupon the contigs where joined. We identified a few short (1-2 kb) regions which were duplicated and hence could be joined to more than two other contigs in the de novo assembly. We resolved positions of these regions by adding them in the plastome assembly as many times as was necessary for all the plastid contigs to be joined in a circle. This methodology worked well for joining all the plastid contigs into a well supported hypothesis for a plastome assembly of species in the refractory clade, deposited on GenBank as unverified whole plastome assemblies. The exception was red clover (Trifolium pratense) for which only a partial assembly could be reliably recovered. The quality of each plastome assembly was verified by visually by inspecting a BWA mem pileup, v. 0.7.5a [28], of paired end reads using Tablet v.1.13.12.17 [29]. We ensured that the connections between manually joined contigs were supported by paired-end read mapping. Finally all plastome assemblies were annotated using DOGMA [11]. Maps of plastid genomes were generated using GenomeVX [30] and visually adjusted using Inkscape (www.inkscape.org).

\section{Identification and analysis of repeated DNA in the plastid genomes}

Repeated segments where determined in each of the plastid genomes by a reciprocal BLASTN search [27], using the NCBI's online BLAST service (http://blast. ncbi.nlm.nih.gov/), where each sequence was used as the query and subject. We used these BLAST outputs to generate dotplots, using R [31]. We decided to analyze repetitive regions that the BLASTN search identified as $300 \mathrm{bp}$ or longer and had an E-value of 0 , by subjecting them to a BLASTX search using a custom database of plastid genes from closely related species. A BLASTX similarity cutoff was set by removing all hits with an Evalue larger than 1E-06. We furthermore subjected all sequences that did not show significant BLASTX similarity to the Trifolium plastid genes, to a BLASTX and BLASTN search to NCBI's nucleotide collection (nt/nr) and non-redundant protein sequences (nr). In order to estimate the percentage of repetitive sequences within the plastomes, we performed a reciprocal BLASTN search of each plastid genome. Only BLAST hits with an alignment length over 79-bp and E-value of 1E-06 were used in the calculations. To gain an overall view of variation in the plastid genomes used in this study, the genomes were aligned using program MAUVE [32]. This alignment is given in Additional file 1.

\section{Phylogenetic analysis}

Due to the extensive rearrangements observed in the plastomes, we restricted our plastome phylogenetic analysis to protein coding genes. We used a custom phylogenetic pipeline, plast2phy, that extracted protein coding regions from DOGMA annotated plastomes, aligned individual gene with Mafft v. 7.0.5 (-auto flag) [33], trimmed alignment gaps using trimAl v.1.2 (-automated1 flag) [34] and finally generated a concatenated alignment of all genes. The pipeline, Plast2phy, written in Python, is available at https://github.com/saemi/plast2phy. We only included genes that were present in all species and showed no evidence of duplication. Models of base substitution were tested for the concatenated matrix using jModelTest v.2.1.1 [35,36]. Using the Akaike information criterion, we determined the GTR + G + I model optimal for the concatenated plastome alignment. We analyzed the dataset under maximum likelihood (ML) [37], using GARLI [38]. We ran GARLI v. 2.0 with default settings, using ten independent searches and 100 bootstrap replicates. Bootstrap consensus was calculated using SumTrees v. 3.3.1 in the DendroPy package [39]. To obtain an approximate age for nodes on this tree by molecular dating we set the Medicago-Trifolium divergence at 24.1 MYA [40] and estimated the other node ages under penalized likelihood [41] implemented in r8s v.1.8 [42]. We chose the penalized likelihood algorithm since a likelihood ratio test, using constrained and unconstrained likelihood scores obtained from PAUP* [43], rejected the molecular clock ( $\mathrm{p}<0.001)$. Confidence intervals were estimated by running dating analysis on 100 bootstrap resampled datasets generated by GARLI and are represented as two times the standard deviation. Trees from phylogenetic analysis were drawn using FigTree v.1.4.0 (http://tree.bio.ed.ac.uk/software/ figtree/), rooted with Medicago truncatula [GenBank: NC_003119] and visually adjusted using Inkscape (http:// www.inkscape.org/).

\section{Availability of supporting data}

The sequence data generated in this study are available in GenBank under the accession numbers KJ788284KJ788292 (see Table 1). The sequence alignment matrix and the corresponding phylogenetic tree are available in the Dryad Digital Repository (doi:10.5061/dryad. km38g) [44]. 


\section{Additional file}

\section{Additional file 1: Genome alignments of plastomes reported in this} paper using MAUVE.

\section{Competing interests}

The authors declare that they have no competing interests.

\section{Authors' contributions}

SS planned the work, performed the data analysis and wrote the manuscript. QC jointly planned the work, co-wrote the manuscript and obtained funding for the study. Both authors read and approved the final manuscript.

\section{Acknowledgements}

The authors are very grateful to the U.S. Department of Agriculture (USDA) National Plant Germplasm System for providing seeds for this study, in particular T. Fields at the Plant Genetic Resources Conservation Unit (PGRCU). We would also like to thank D. Kaplan for greenhouse assistance, D. Huang for good tips for data analysis, and A. Kuzmin for assistance in the preparation of sequencing libraries. Finally we thank UBC and NSERC for funding.

Received: 9 May 2014 Accepted: 27 October 2014

Published online: 18 November 2014

\section{References}

1. Moore MJ, Dhingra A, Soltis PS, Shaw R, Farmerie WG, Folta KM, Soltis DE: Rapid and accurate pyrosequencing of angiosperm plastid genomes. BMC Plant Biol 2006, 6:17

2. Wicke S, Schneeweiss GM, de Pamphilis CW, Müller KF, Quandt D: The evolution of the plastid chromosome in land plants: gene content, gene order, gene function. Plant Mol Biol 2011, 76:273-297.

3. Guisinger MM, Kuehl J, Boore $J \mathrm{~L}$, Jansen RK: Extreme reconfiguration of plastid genomes in the angiosperm family Geraniaceae: rearrangements, repeats, and codon usage. Mol Biol Evol 2011, 28:583-600.

4. Haberle RC, Fourcade HM, Boore JL, Jansen RK: Extensive rearrangements in the chloroplast genome of Trachelium caeruleum are associated with repeats and tRNA genes. J Mol Evol 2008, 66:350-361.

5. Ištvánek J, Jaroš M, Křenek A, Řepková J: Genome assembly and annotation for red clover (Trifolium pratense; Fabaceae). Am J Bot 2014, 101:327-337.

6. Yates SA, Swain MT, Hegarty MJ, Chernukin I, Lowe M, Allison GG, Ruttink T, Abberton MT, Jenkins G, Skøt L: De novo assembly of red clover transcriptome based on RNA-Seq data provides insight into drought response, gene discovery and marker identification. BMC Genomics 2014 $15: 453$.

7. Cai Z, Guisinger M, Kim H-G, Ruck E, Blazier JC, McMurtry V, Kuehl JV, Boore J, Jansen RK: Extensive reorganization of the plastid genome of Trifolium subterraneum (Fabaceae) is associated with numerous repeated sequences and novel DNA insertions. J Mol Evol 2008, 67:696-704.

8. Wojciechowski MF, Sanderson MJ, Steele KP, Liston A: Molecular phylogeny of the "temperate herbaceous tribes" of papilionoid legumes: a supertree approach. In Advances in Legume Systematics, Part 9. Edited by Herendeen PS, Bruneau A. Kew, UK: Royal Botanic Gardens; 2000:277-298.

9. Magee AM, Aspinall S, Rice DW, Cusack BP, Sémon M, Perry AS, Stefanović S, Milbourne D, Barth S, Palmer JD, Gray JC, Kavanagh TA, Wolfe KH: Localized hypermutation and associated gene losses in legume chloroplast genomes. Genome Res 2010, 20:1700-1710.

10. Sabir J, Schwarz E, Ellison N, Zhang J, Baeshen NA, Mutwakil M, Jansen R, Ruhlman T: Evolutionary and biotechnology implications of plastid genome variation in the inverted-repeat-lacking clade of legumes. Plant Biotechnol J 2014, doi:10.1111/pbi.12179.

11. Wyman SK, Jansen RK, Boore JL: Automatic annotation of organellar genomes with DOGMA. Bioinformatics 2004, 20:3252-3255.

12. Ellison NW, Liston A, Steiner JJ, Williams WM, Taylor NL: Molecular phylogenetics of the clover genus (Trifolium - Leguminosae). Mol Phylogenet Evol 2006, 39:688-705.

13. Cosner ME, Jansen RK, Palmer JD, Downie SR: The highly rearranged chloroplast genome of Trachelium caeruleum (Campanulaceae): multiple inversions, inverted repeat expansion and contraction, transposition, insertions/deletions, and several repeat families. Curr Genet 1997, 31:419-429.

14. Chumley TW, Palmer JD, Mower JP, Fourcade HM, Calie PJ, Boore JL, Jansen RK: The complete chloroplast genome sequence of Pelargonium $x$ hortorum: organization and evolution of the largest and most highly rearranged chloroplast genome of land plants. Mol Biol Evol 2006, 23:2175-2190

15. Lilly JW, Havey MJ, Jackson SA, Jiang J: Cytogenomic analyses reveal the structural plasticity of the chloroplast genome in higher plants. Plant Cell 2001, 13:245-254.

16. Maréchal A, Parent J-S, Véronneau-Lafortune F, Joyeux A, Lang BF, Brisson N: Whirly proteins maintain plastid genome stability in Arabidopsis. ProC Natl Acad Sci U S A 2009, 106:14693-14698.

17. Maréchal A, Brisson N: Recombination and the maintenance of plant organelle genome stability. New Phytol 2010, 186:299-317.

18. Cerutti H, Osman M, Grandoni P, Jagendorf AT: A homolog of Escherichia coli RecA protein in plastids of higher plants. Proc Natl Acad Sci U S A 1992, 89:8068-8072.

19. Cerutti $H$, Jagendorf AT: DNA strand-transfer activity in pea (Pisum sativum L.) chloroplasts. Plant Physiol 1993, 102:145-153.

20. Kode V, Mudd EA, lamtham S, Day A: The tobacco plastid accD gene is essential and is required for leaf development. Plant J 2005, 44:237-244.

21. Gurdon C, Maliga P: Two distinct plastid genome configurations and unprecedented intraspecies length variation in the accD coding region in Medicago truncatula. DNA Res 2014, 21:1-11.

22. Rousseau-Gueutin M, Huang $X$, Higginson E, Ayliffe M, Day A, Timmis JN: Potential functional replacement of the plastidic acetyl-CoA carboxylase subunit $(\mathrm{accD})$ gene by recent transfers to the nucleus in some angiosperm lineages. Plant Physiol 2013, 161:1918-1929.

23. Maul JE, Lilly JW, Cui L, de Pamphilis CW, Miller W, Harris EH, Stern DB: The Chlamydomonas reinhardtii plastid chromosome: islands of genes in a sea of repeats. Plant Cell 2002, 14:2659-2679.

24. Mackenzie S, Mclntosh L: Higher plant mitochondria. Plant Cell 1999, 11:571-585.

25. Doyle J, Doyle JL: A rapid DNA isolation procedure for small quantities of fresh leaf tissue. Phytochem Bull 1987, 19:11-15.

26. Lohse M, Bolger AM, Nagel A, Fernie AR, Lunn JE, Stitt M, Usadel B: RobiNA: a user-friendly, integrated software solution for RNA-Seq-based transcriptomics. Nucleic Acids Res 2012, 40:W622-W627.

27. Zhang Z, Schwartz S, Wagner L, Miller W: A greedy algorithm for aligning DNA sequences. J Comput Biol 2000, 7:203-214.

28. Li H, Durbin R: Fast and accurate short read alignment with Burrows-Wheeler transform. Bioinformatics 2009, 25:1754-1760.

29. Milne I, Stephen G, Bayer M, Cock PJ, Pritchard L, Cardle L, Shaw PD, Marshall D: Using tablet for visual exploration of second-generation sequencing data. Brief Bioinform 2013, 14:193-202

30. Conant GC, Wolfe KH: GenomeVx: simple web-based creation of editable circular chromosome maps. Bioinformatics 2008, 24:861-862.

31. R Core Team. R: A language and environment for statistical computing. In Vienna, Austria: R Foundation for Statistical Computing; 2014. http://www.R-project.org/.

32. Darling AE, Mau B, Perna NT: Progressivemauve: multiple genome alignment with gene gain, loss and rearrangement. PLoS One 2010, 5(6):e11147.

33. Katoh K, Standley DM: MAFFT multiple sequence alignment software version 7: improvements in performance and usability. Mol Biol Evol 2013, 30:772-780

34. Capella-Gutiérrez S, Silla-Martínez JM, Gabaldón T: trimAl: a tool for automated alignment trimming in large-scale phylogenetic analyses. Bioinformatics 2009, 25:1972-1973.

35. Guindon S, Gascuel O: A simple, fast, and accurate algorithm to estimate large phylogenies by maximum likelihood. Syst Biol 2003, 52:696-704.

36. Darriba D, Taboada GL, Doallo R, Posada D: jModelTest 2: more models, new heuristics and parallel computing. Nat Methods 2012, 9:772.

37. Felsenstein J: Maximum likelihood and minimum-steps methods for estimating evolutionary trees from data on discrete characters. Syst Zoo 1973, 22:240-249.

38. Zwickl DJ: Genetic Algorithm Approaches for the Phylogenetic Analysis of Large Biological Sequence Datasets under the Maximum Likelihood Criterion, Dissertation. Austin, TX: University of Texas; 2006.

39. Sukumaran J, Holder MT: DendroPy: a python library for phylogenetic computing. Bioinformatics 2010, 26:1569-1571 
40. Lavin M, Herendeen PS, Wojciechowski MF: Evolutionary rates analysis of Leguminosae implicates a rapid diversification of lineages during the tertiary. Syst Biol 2005, 54:575-594.

41. Sanderson MJ: Estimating absolute rates of molecular evolution and divergence times: a penalized likelihood approach. Mol Biol Evol 2002, 19:101-109.

42. Sanderson MJ: r8s: inferring absolute rates of molecular evolution and divergence times in the absence of a molecular clock. Bioinformatics 2003, 19:301-302.

43. Swofford DL: PAUP*. Phylogenetic Analysis Using Parsimony (*and Other Methods), Version 4. Sunderland, Massachusetts: Sinauer Associates; 2003.

44. Sveinsson S, Cronk Q: Data from: evolutionary origin of highly repetitive plastid genomes within the clover genus (Trifolium). In Dryad Digital Repository. doi:10.5061/dryad.km38g.

doi:10.1186/s12862-014-0228-6

Cite this article as: Sveinsson and Cronk: Evolutionary origin of highly repetitive plastid genomes within the clover genus (Trifolium). BMC Evolutionary Biology 2014 14:228.

\section{Submit your next manuscript to BioMed Central and take full advantage of:}

- Convenient online submission

- Thorough peer review

- No space constraints or color figure charges

- Immediate publication on acceptance

- Inclusion in PubMed, CAS, Scopus and Google Scholar

- Research which is freely available for redistribution 